\title{
Controlling the Security Objects Properties for Networks in Windows 2000
}

Dhuha Basheer Abdullah

Abd- Alnaseer Ahmed

Prof.dhuha_basheer@uomosul.edu.iq

College of Computer Sciences and Mathematics

Received on: 27/10/2002

University of Mosul, Iraq

\section{ABSTRACT}

Information security occupies a wide area in designing network operating systems. Windows 2000 was of the operating systems that offered great interest for network security, so it is recently considered as the most important network operating system because of the high extended capabilities in network security embedded in it. This search is devoted to the study of the network security in windows 2000. A special program for controlling the security properties for the main objects of the Windows 2000 database (Active Directory) has been built. ActiveX Data Object (ADO) is used for searching the Active Directory and ADSI interface for accessing security principal accounts. This software presents the likelihood of accessing the security properties of these objects to the level of flags and bits in the data structure of security properties elements.

Keywords: Operating System, Windows2000 system ,Networks Security, Security Objects

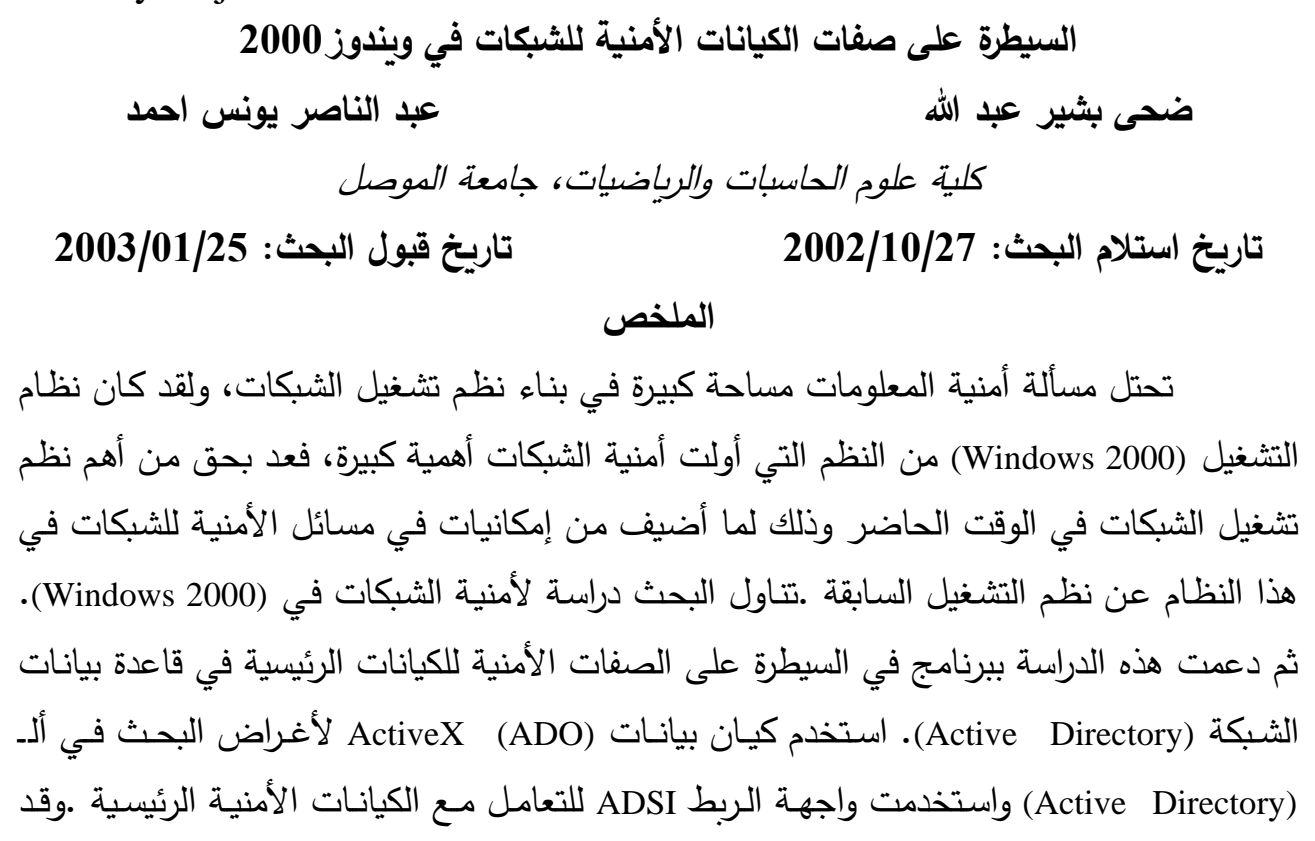




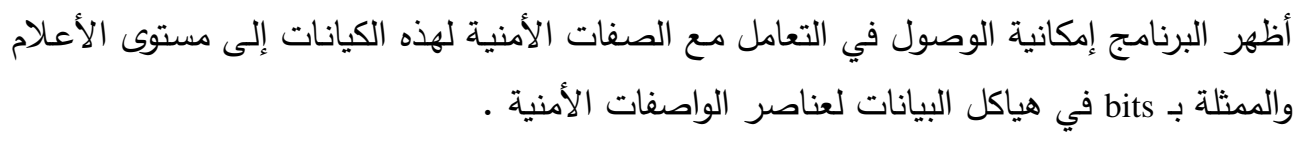
الكلمات المفتاحية: نظام التشغيل، نظام 2000 لـ Windows)، أمنية الثبكات، الكيانات الأمنية.

لا يمكن لأي فرد أو مؤسسة العيش بثكل منعزل،في هذا العصر الذي اصبح العالم فيه

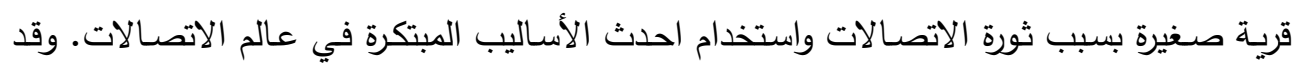

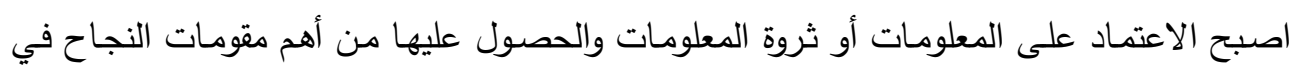
ظل هذا العصر الذي تثكل فيه المعلومات وحداثتها أهم المرتكزات للتقدم والنجاح. إن تبادل هذه التهات

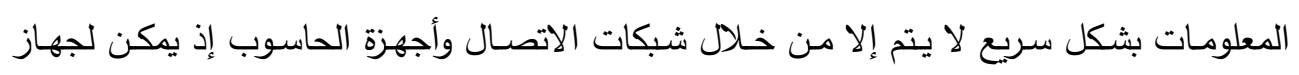
الحاسوب التعامل مع هذه البيانات وعرضها بشكل لآلئل مفهوم. ويمكن تلخيص الفوائد التي تقدمها الثبكات بما يأتي:

1-مشاركة الموارد والملفات (Sharing resources and files)

2-مشاركة البرمجيات التطبيقية (Sharing Applications)

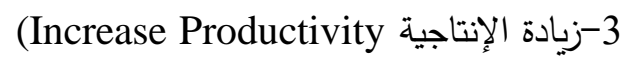

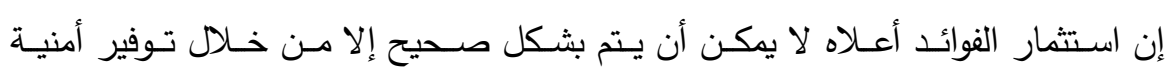

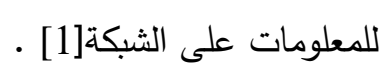

إنَ أمنية الحاسوب تعريف واسع يثمل منع أي وصول غير مسموح أو غير مرخص لأي

جزء من نظام الحاسوب ـ ويندرج في ذلك المعنى جميع جوانب الأجزاء الصلبة (Hardware)

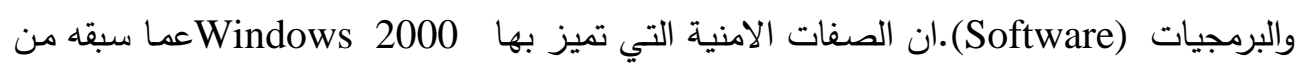
إصدارات Windows هي [5]: 1- جهز بـ مerberos مدكناً التوقيع المفرد للوصول إلى مصادر الثبكة. 2- دعمه نظام تشفير الملفات (EFS).

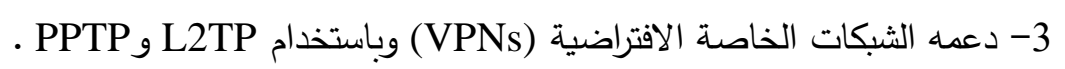
4- جهز بمدير شهادات المفتاح العام Public Key (PK) Certificate manager لغرض إدارة المفتاح. 5- جهز بـ Internet Protocol Security (IPSec) طبقة النقل (Transport Layer). 
6- إمكانيـة إعـداد مراقبـة الحسادث الأمنيـة إلى مسـويات تفصيلية، وتسـجيل الأحـداث لغـرض استعراضها فيما بعد.

7- مستوى عـالٍ من إعدادات التحكم بالوصول إلى مصـادر الثبكة باستخدام المجاميع ، حقوق المستخدم، السماحات ، أو السياسة الأمنية.

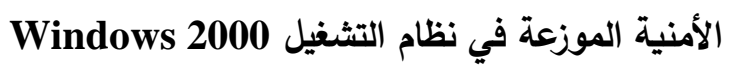

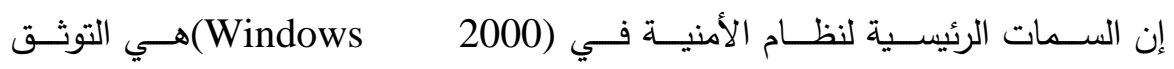

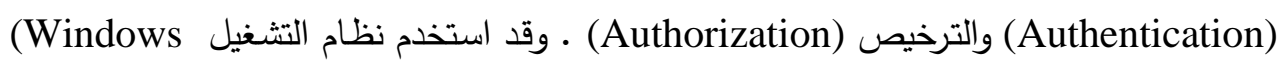

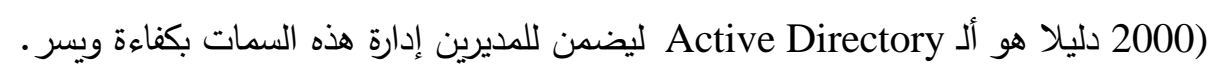

التوثق(Authentication)

التوثق هو عملية التحقق من إن المستخدم الذي يحمل اسما معينا هو نفسه الذي يشير

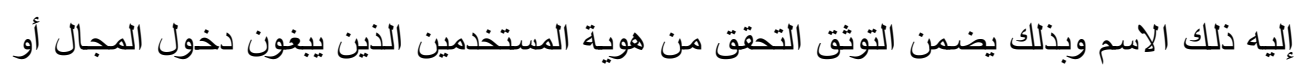

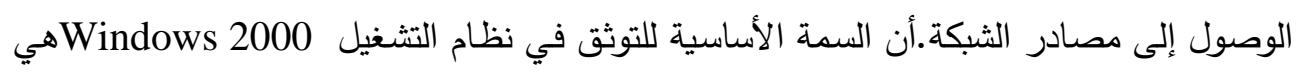

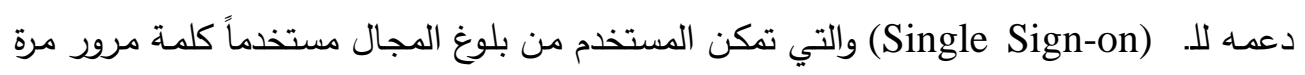
واحدة ويتوثق بعدها إلى أي حاسوب في المجال.

أن عملية التوثق في 2000 Windows تتفذ بوصفها عملية من جزأين: البلوغ التفاعلي وتوثق الشبكة (Interactive Logon)

$$
\text { المستخدم يعتد على الجزأين كليهما [10]. }
$$

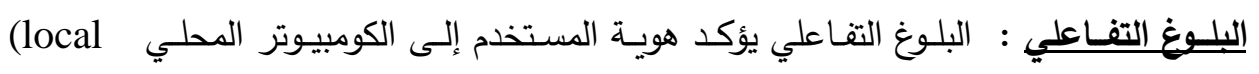

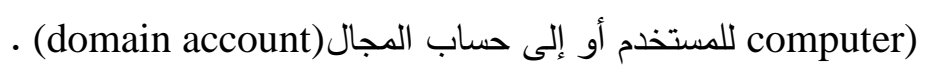

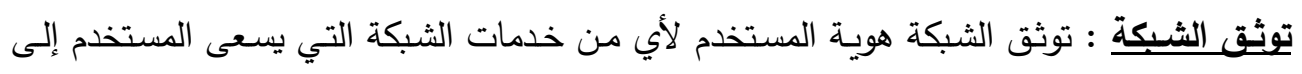

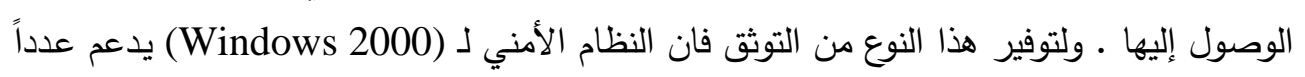

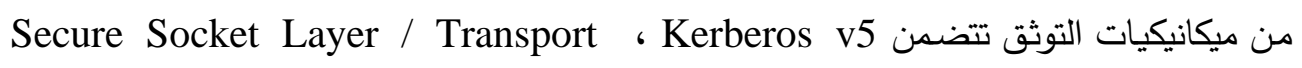

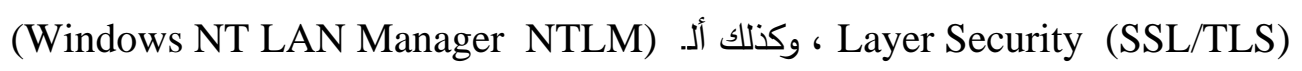
والتي يدعمها لغرض التوافق مع (Windows NT 4.0) [11] [11].

بروتوكول التوثق Kerberos

يدعم (Windows 2000) مجموعة بروتوكولات للتحقق من هويـة المستخدمين الذين

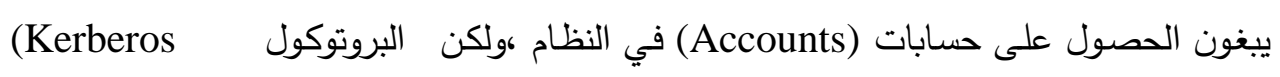




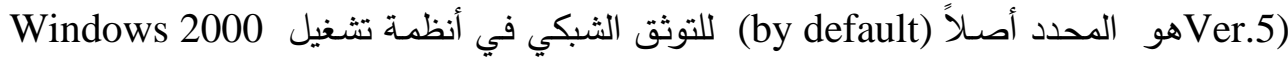

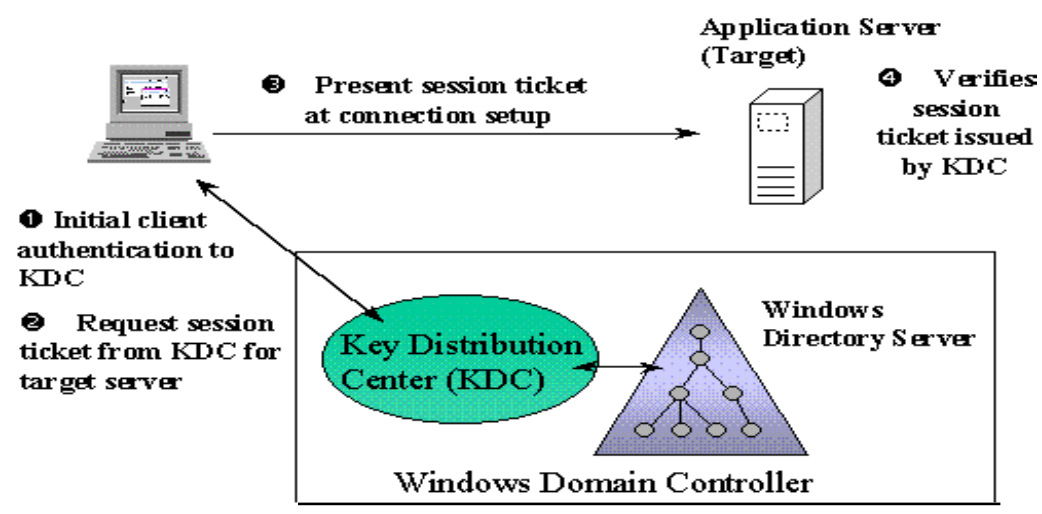

Kerberos الثكل(1):مخطط عام للبروتوكول

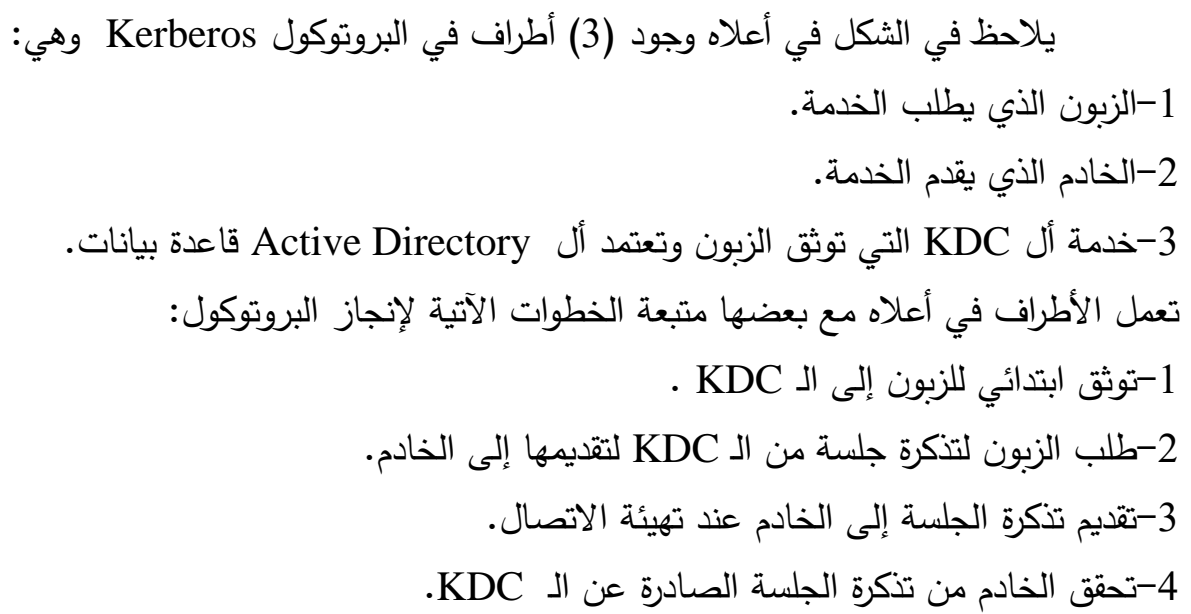

\section{Authorization}

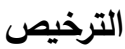

الترخيص هو العملية التي تثبت أن للمستخدم الحقوق الصحيحة والسماحات للوصول إلى هـ

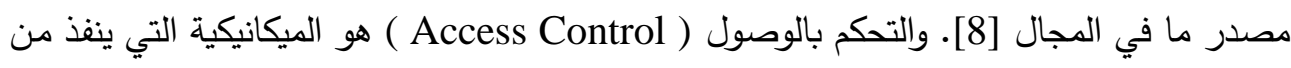

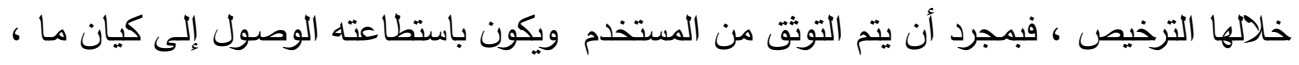


فان نوع الوصول الممنوح يحدد إما عن طريق حقوق الاستخدام المخصصة للمستخدم أو عن طريق

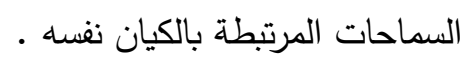

عناصر نموذج التحكم بالوصول

يتكون نموذج التحكم بالوصول من عنصرين رئيسيين:

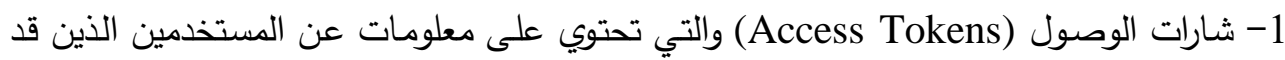

تم بلوغهم الثبكة (Logged-on Users).

2- الواصفات الأمنية (Security Descriptors) والتي تحتوي على معلومات أمنية تحمي الكيان

.[8] [3] [2]

شارات الوصول (Access Tokens)

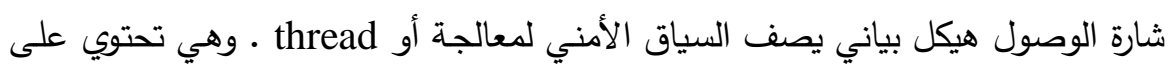

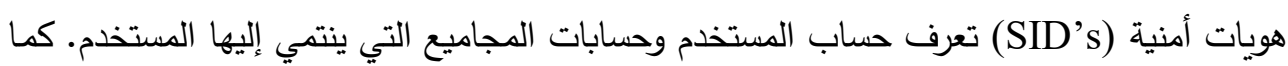

تحتوي على قائمة بالامتيازات التي يتمتع بها المستخدم أو مجموعته الأمنية.

(Security Descriptor) الواصفات الأمنية

تحتوي الواصفات الأمنية على معلومات التحكم بالوصول والمتعلقة بكيان ما ـ والواصف

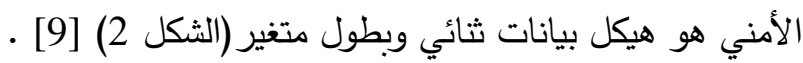

\begin{tabular}{|c|}
\hline Header \\
\hline Owner SID \\
\hline Group SID \\
\hline DACL \\
\hline SACL \\
\hline
\end{tabular}

الثكل (2) الهيكل البياني للواصف الأمني

وعندما يحـاول المستخدم القيـام بأي فعل مـن الأفعـال الممكنـة على الكيان فـإن نظـام

التشغيل يقوم بفحص الواصف الأمني للكيان وذلك لتحديد فيما إذا كان مسموحاً للمستخدم القيام بالفعل الذي يريده . 


$$
\text { إن المكونات الرئيسية للواصف الأمني هي : }
$$

ت Header تصف الميزات الخاصة بالواصف الأمني • • Owner : Primary Group الحكر : Access Control Lists التحكم بالوصول (Access Control Entries ACE’s) التي تحدد الحماية المستخدمة لكيان

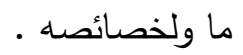
ويمكن للواصف الأمني أن يحتوي على نوعين من أل ACL : :1- قائُة التحكم بالوصول الاستتسابي (Discretionary Access Control List DACL) التي تحدد المستخدمين والمجموعات المسموح لها والممنوعة من الوصول الوستئل 2- قائمسة النظام للتحكم بالوصول (System Access Control List SACL) التي تبين الكيفية التي يتم بها تسجيل عملية الوصول ـ والثكل (3) يوضـح الهيكل البياني لقائمسة التحكم بالوصول.

\begin{tabular}{|l|l|}
\hline ACL Size & ACL Revision \\
\hline ACL Count & \\
\hline ACE $[1]$ & $1]$ \\
\hline ACE $[\ldots]$ & $\ldots$ ACE $[n]$ \\
\hline
\end{tabular}

الثكل (3 ) قائمة التحكم بالوصول

مداخل التحكم بالوصول ( Access Control Entries ACEs ) إن جميع مداخل التحكم بالوصول (ACEs) تحتوي على معلومات التحكم بالوصول الأتية :يعرف هوية مستخدم أو مجموعة. SID يعين حقوق الوصول مع (access mask) - مجموعة من الأعلام (bit flags) تحدد إمكانية وراثة كيانات الابن لا ACE وعلم يثلم يثير إلى نوع - أل 
يدعم (Windows 2000) ستة أنواع من مداخل التحكم بالوصول ـ ثلاثة منها من نوع

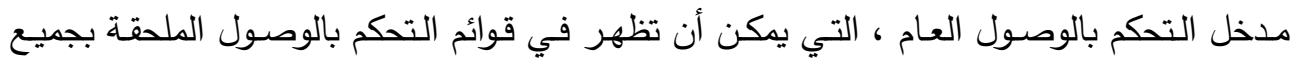

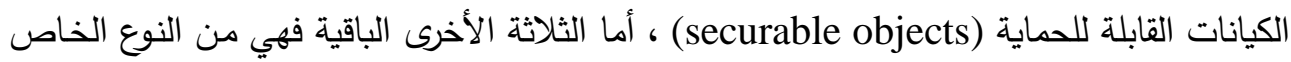
بكيانات خاصة (object-specific) التي يمكن أن توجد فقط في قوائم التحكم بالوصول لكيانات • [9] (Active Directory) والثكلان (4) و (5) يوضحان نوعي مداخل التحكم بالوصول.

\begin{tabular}{|c|c|}
\hline ACE Size & ACE Type \\
\hline Inheritance and Audit Flag & \\
\hline Access Mask & \\
\hline
\end{tabular}

الثكل (4) مدخل التحكم بالوصول العام

\begin{tabular}{|c|c|}
\hline ACE Size & ACE Type \\
\hline \multicolumn{2}{|l|}{ Inheritance and Audit Flags } \\
\hline \multicolumn{2}{|l|}{ Access Mask } \\
\hline Object Type & Inherited Object Type \\
\hline
\end{tabular}

الثكل (5) مدخل التحكم بالوصول الخاص بالكيانات

Active Directory الاليل النشط

ألـ (Active Directory) وهو خدمة دليل لـ (Windows 2000 Server) يخزن

معلومات حول كيانات موجودة على الثبكة ويسهل على المدراء والمستخدمين إيجادها واستخدامها .

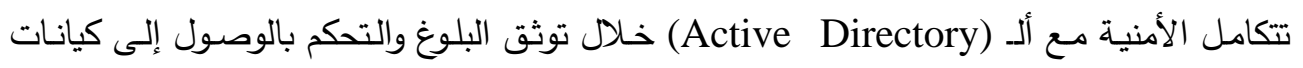

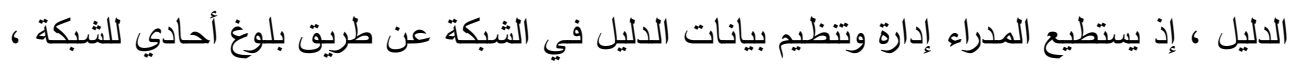

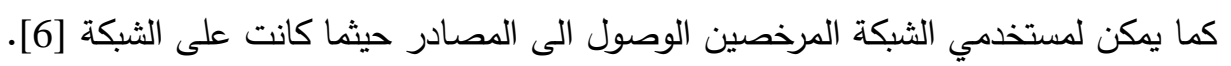

خواص ألـ (Active Directory) وفوائده يتضمن أل (Active Directory) الخواص والفوائد الأتية : - التحكم بالوصول نزولا الى مستوى الصفات للحسابات. 
- الإدارة المرتكزة على السياسة ( Policy - Based Demonstration ) - يخزن ألـ (Active Directory) السياسات التي تدعى كيانات ( Group Policy ) والتي ( تخصص كل منها لسياق معين . تخدان - قابلية التوسع. - قابلية العمل البيني مع خدمات دليل أخرى. - الدعم لصيغ الاسم القياسي لضمان سهولة النقل من نظام إلى آخر وسهولة الاستخدام. - توفر مجموعة غنية من أل (Application Programming Interfaces APIs).

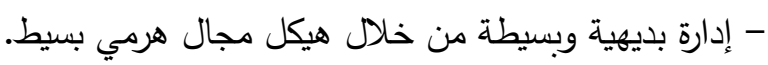

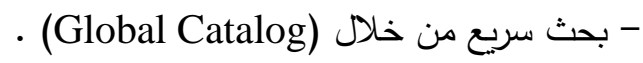
- تحديث سريع ومتوافق من خلال توحيد النسخ متعدد الأسياد .

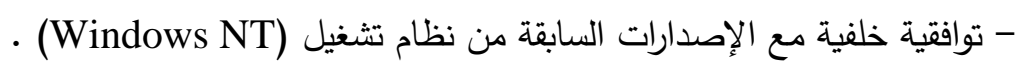

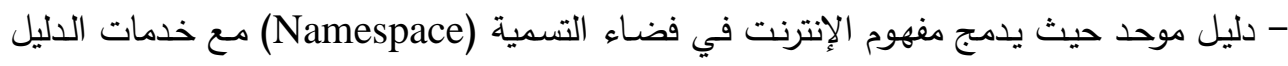
لنظام التشغيل.

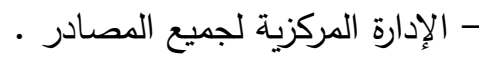
- توفر ألـ ( Active Directory Service Interface ( ADSI لتسهيل البرمجة والتعامل مع ألـ وأداء مهام إدارية شائعة كإضافة مستخدمين جدد ، إدارة الطابعات وتحديد (Active Directory)

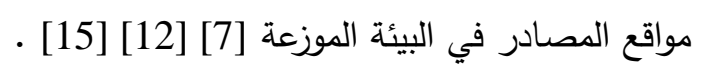

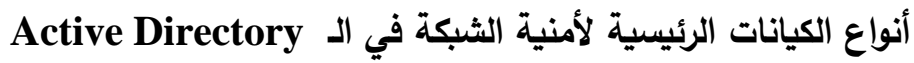

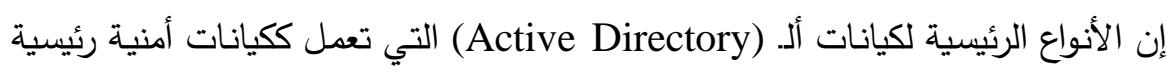
[14]: هي

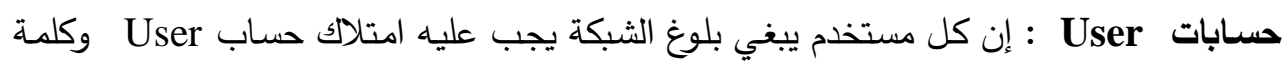
مرور خاصة به . حسابات Computer وهي حسابات تحدد أي الحواسيب الزبائن هي أعضاء في المجالات المعينة.

حسـابات Groups : هنالك نوعان رئيسان من المجموعات (Groups) : مجموعات Security حسابات User حماب 
تكوين البرنامج

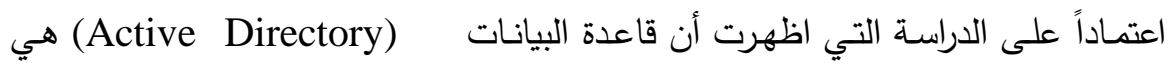
العنصر الأهم في مسائل إدارة الثبكة والتي من ضمنها مسألة أمنية الثبكة تم في هذا البحث بناء

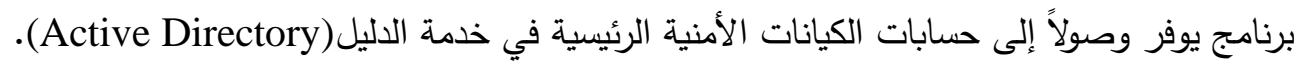

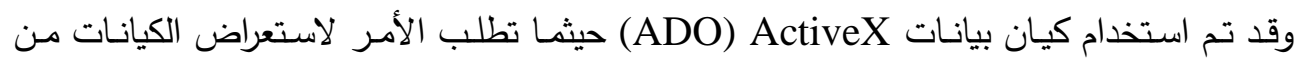

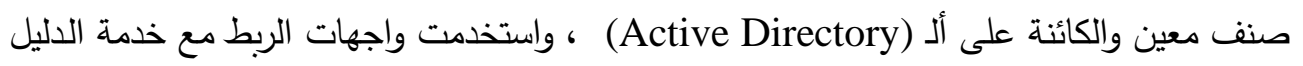

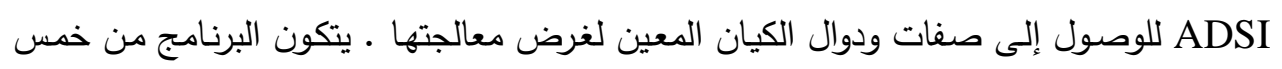

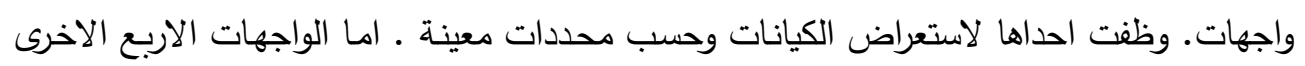

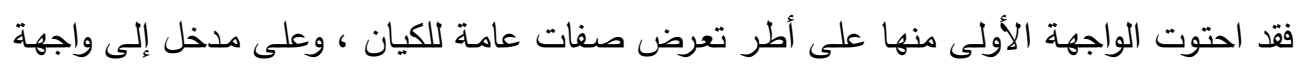

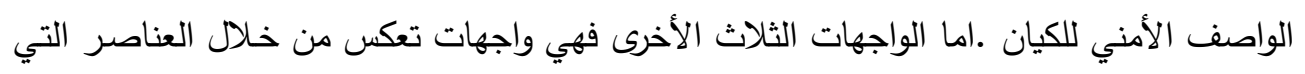

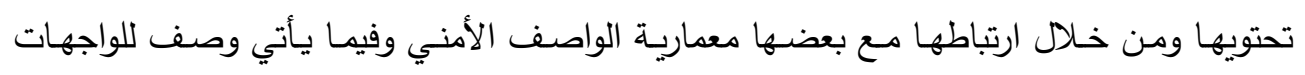
المكونة للبرنامج.

واجهة استعراض الكيانات (الثكل 5 )

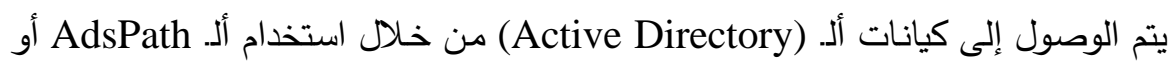
ألـ GUID في عملية الربط إلى الكيان في واجهات اتصــال لى ADSI. تحتوي واجهة اتصـال استعراض الكيانات على عناصر عديدة ـ ففيما يتعلق بصنف الكيان فإن واجهة الاتصال تحتوي

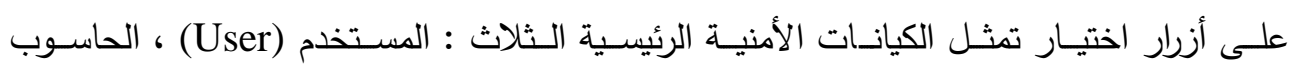

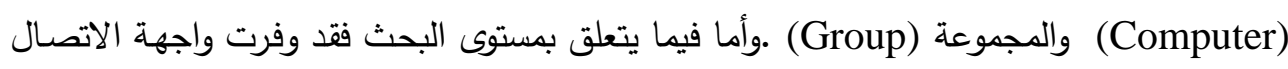
ثلاثة مستويات للبحث : القاعدة (Base) ، مستوى واحد (One Level) والثجرة الثانوية (Sub)

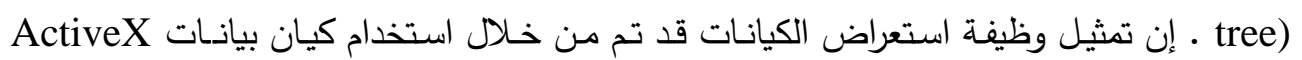

$$
\text { وعلى وفق الخطوات الأتية :- (ADO) }
$$

1- تكوين كيان ADOConnection

- تخصيص قيمـة لصفة ADsDS Object هين Aي :Connection::Provider:

- Active Directory 
Connection::Open تأسيس ربط فيزيائي إلى مصدر البيانات ويتم ذلك من خلال استخدام Active Directory هوأسم مصدر البيانات في حالة أل. Active Directory.method . "Provider

\section{2- Connection :Command تكوين كيان}

يعرف كيان Command بأنـه أمر معين في النية استخدامه في التنفيذ على مصدر

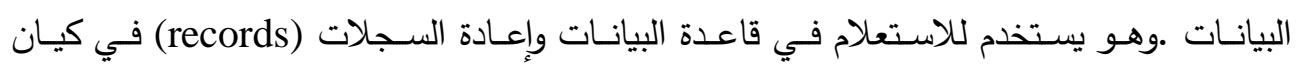

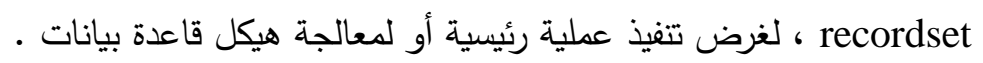
3- قراءة ألـ AdsPath للمجال الذي سيتم البحث فيه. 4- بناء عنصر Commandtext

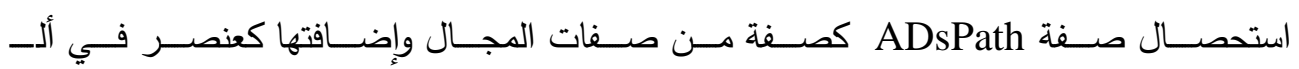
Commandtext بناء عنصر المرشح من أل Commandtext.

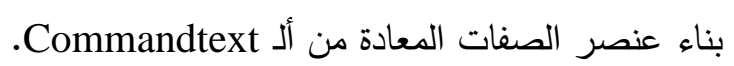

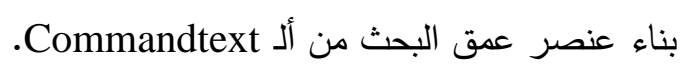

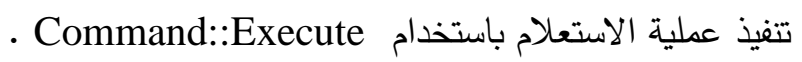

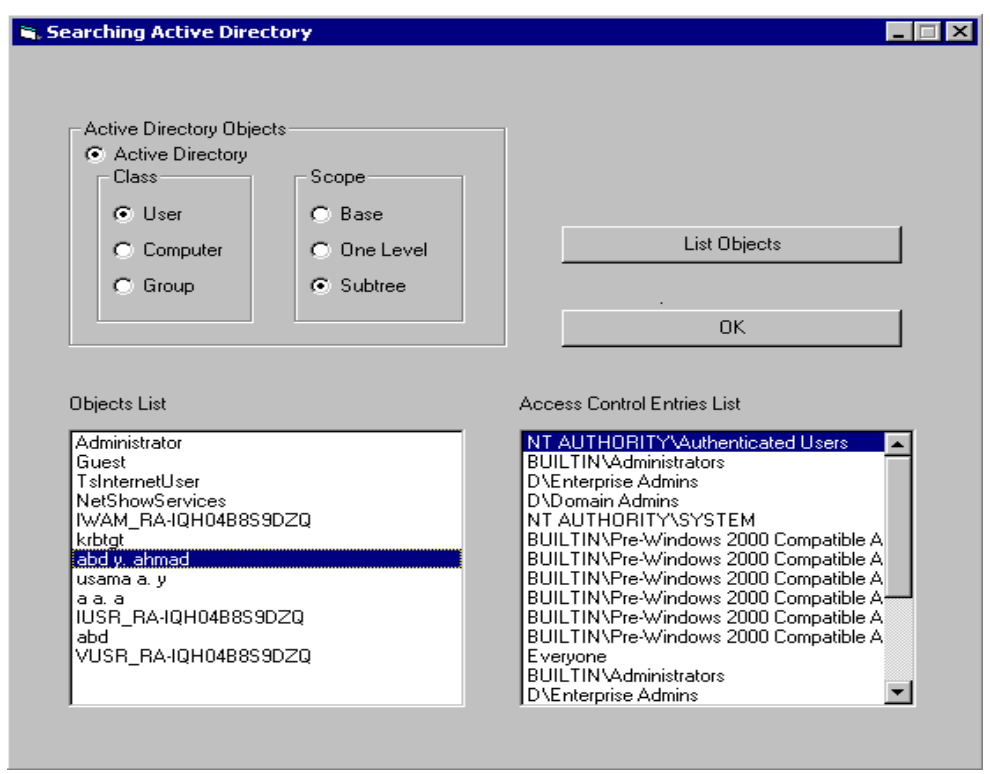

الثكل(5)واجهة استعراض الكيانات 
واجهة الصفات العامة للكيان Main Properties (الثكل 6)

إن عناصر هذه الواجهة هي :

- أطر الصفات العامة :

تعرض في هذه الاطر صفات عامـة للكيانـات التي يتم اختيارهـا من خـلال واجهـة استعراض

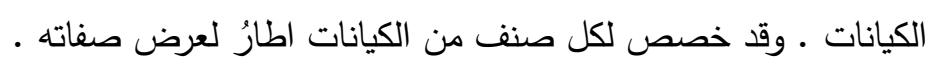

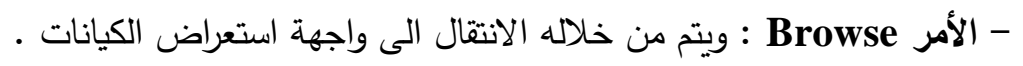

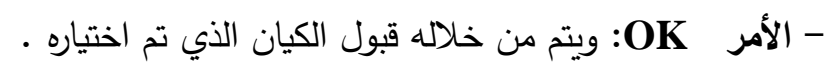

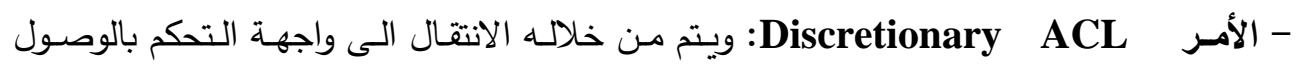

الاستتسابي - (الامتر

- الأمر Security Descriptor : ويتم من خلاله الانتقال الى واجهة الواصف الامني .

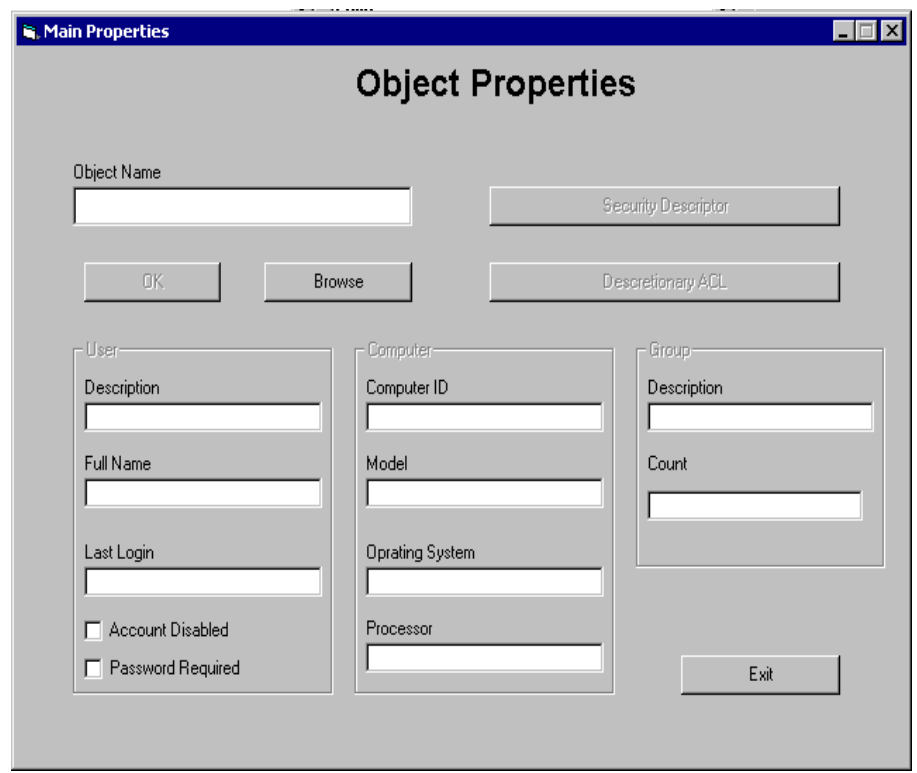

الثكل (6) واجهة الصفات العامة للكيان 


\section{واجهة الواصف الأمني Security Descriptor (الثكل7)}

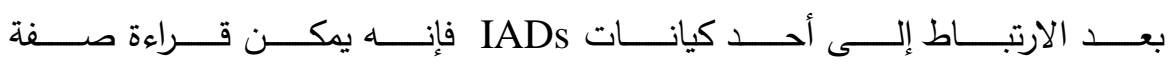

"ntSecurityDescriptor" كيان"IADsSecurity Descriptor" والذي بدوره يتكون من مجموعة من الصفات والدوال.

$$
\text { وتتكون واجهة الواصف الأمني من العناصر الاتية : }
$$

- صندوق النص Revision : ويحتوي على قيمة Revision للواصف الأمنية : الأني ـ وهذه القيمة

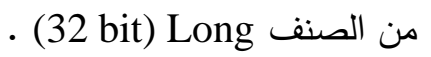
- صندوق النص Owner : ويمثل النص فيه اسم المالك (Owner) للكيان .

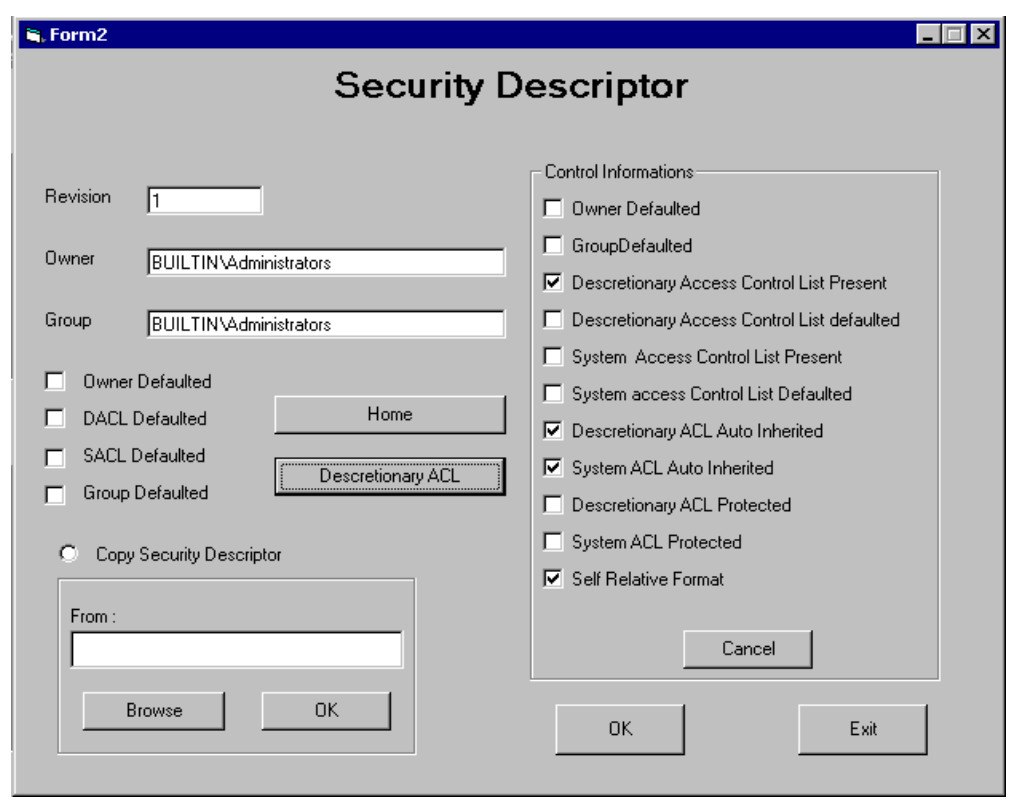

$$
\text { الثكل(7): إجهة الواصف الامني }
$$

- صندوق النص Group : ويمثل النص فيه أسم المجموعة ( Group ) التي تنتمي إليها الهوية

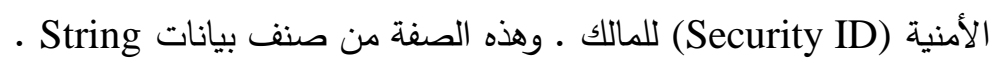

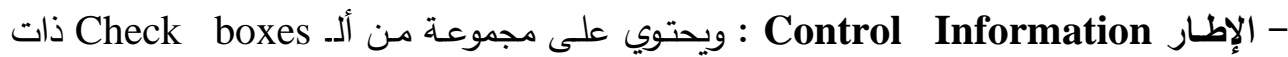

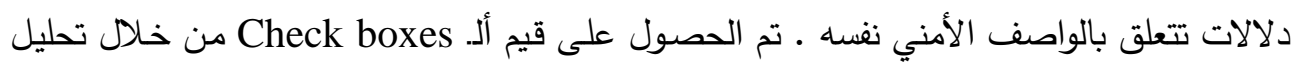

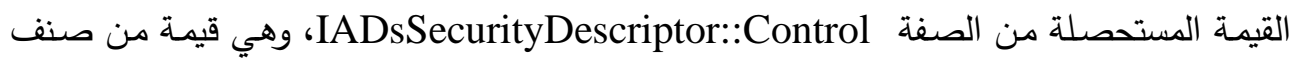




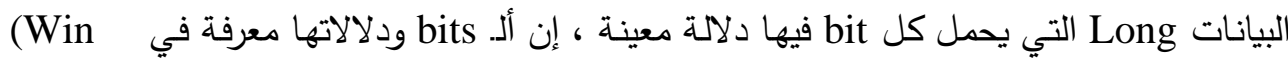
. structure 32 Security _ Descriptor _ Control) الإطار Copy Security Descriptor : إذ يمكن من خلال التعامل مع عناصر هذا الإطار

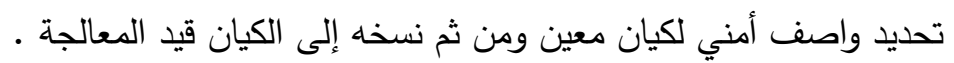

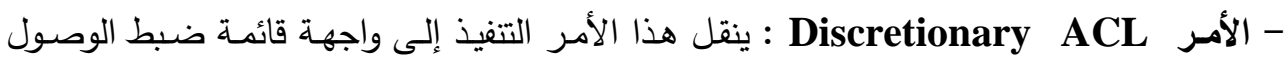
الاستتسابي ليتم من خلالها عرض محتويات قائمة الوصول وأداء وظائف معينة تتعلق بهذه القائمة

$$
\text { تتم هذه العملية من خلال الخطوات آلاتية : }
$$

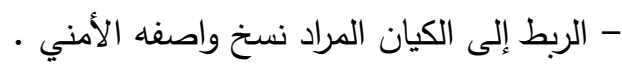

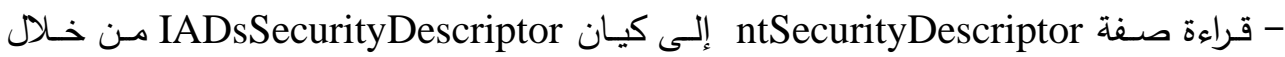
دالة القراءة :IADs::Get. - اســتخدام الدالـة IADsSecurityDescriptor::CopySecurityDescriptor لنســخ هــهـ الصفة إلى كيان من نوع IADsSecurityDescriptor - إحـال الكيان الجديد في صفة ntSecurityDescriptor للكيان قيد المعالجـة باستخدام دالة . IADs::put الكتابة - تبيت التغير الحاصل إلى أل Active Directory باستخدام الدالة IADs::Set Info -

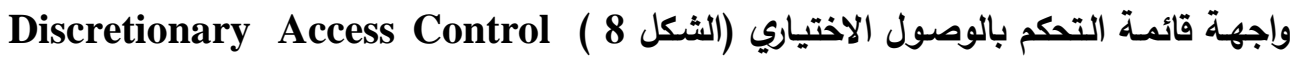

إن جميع الصفات والدوال التي استخدمت في الوصول إلى ومعالجة بيانات قائمة التحكم

بالوصول الاختياري هي صفات ودوال لواجهة الربط الأمنية IADsAccessControlList إن إن الإجراء العام الذي تم لإدارة سيطرة الوصول على كيان IADs تطلب الخطوات الأتية :

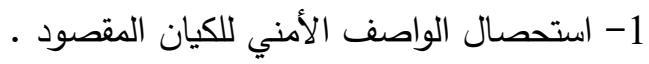

2- استحصال قائمة الوصول الأختياري من الواصف الأمني .

3- معالجة مداخل التحكم بالوصول في قائمة الوصول الأختياري .

$$
\text { إن عناصر واجهة قائمة الوصول الأختياري هي : }
$$


- صندوق النص ACL Revision: تمت قراءة قيمة Revision لقائمة الوصول الأختياري من خلال دالة الصفة IADsAccessControlList::AclRevision .إن صنف البيانات لهذه القيمة هو Long ـ وتمثل هذه القيمة مستوى أل الـ Revision لقائمة الوصول.

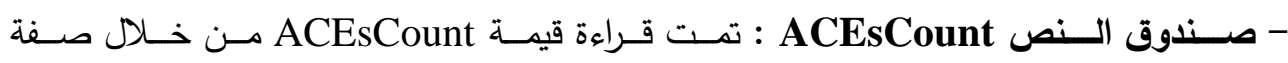
إن صنف البيانات لهذه القيمة هو IADsAccessControlList::AclCount هذه القيمة عدد مداخل التحكم بالوصول في قائمة التحكم بالوصول .

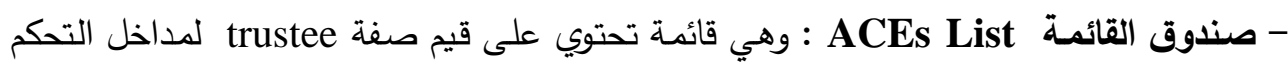
بالوصول في قائمة التحكم بالوصول . - الإطار Copy ACEs To List يتم من خلال هذا الإطار اختيار كيان معين ونسخ

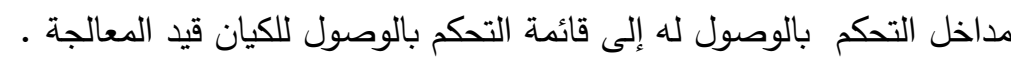

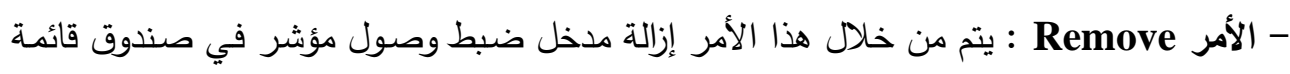

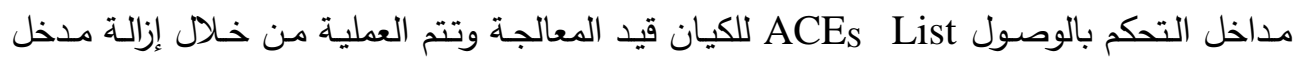
التحكم بالوصول من خلال استخدام الدالة IADsAccessControlList:RemoveAce. - الأمر Display : يتم من خلال هذا الأمر فتح واجهة خاصـة ببيانات ووظائف مدخل التحكم

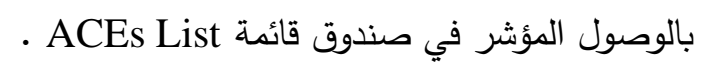
- الأمر Add Exist: يتم من خلال هذا الأمر إضافة مدخل تحكم بالوصول يعود إلى كيان موجود في الـ Active Directory إلى قائمة التحكم بالوصول للكيان قيد المعالجة .

IADsAccessControl List::Add Ace.

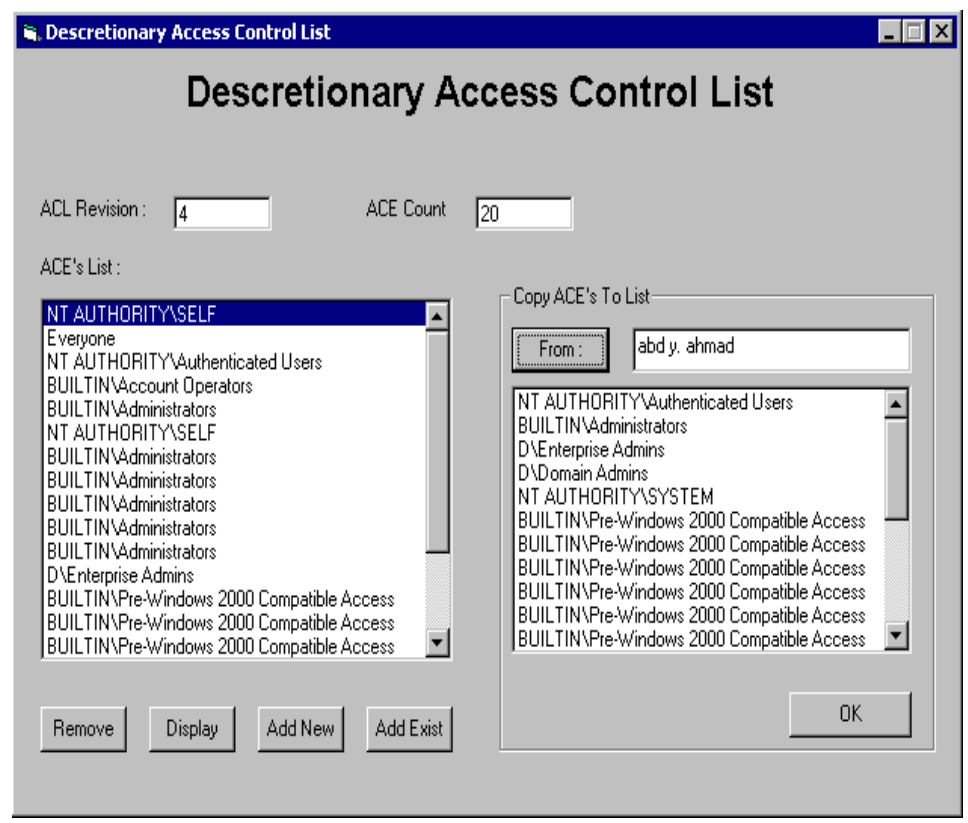

الثكل (8) : قائمة التحكم بالوصول الاختياري 
واجهة مدخل التحكم بالوصول Access Control Entry (الثكل 9)

إن صفات مدخل التحكم بالوصول يمكن الوصول إليها ومعالجتها من خلال دوال الصفات

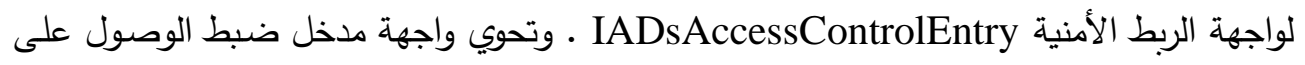

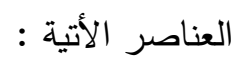

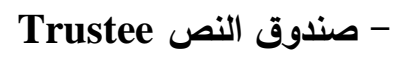

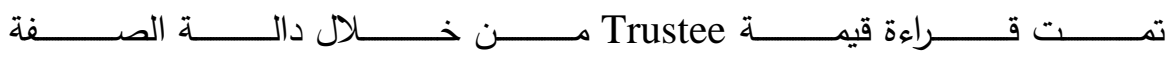

IADsAccessControlEntry::Trustee الكيان المنوح سماحات الوصول المثبتة في مدخل التحكم بالوصول .

- صندوق النص Object Type -

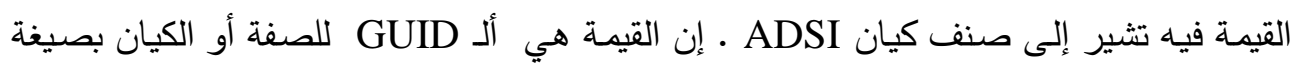

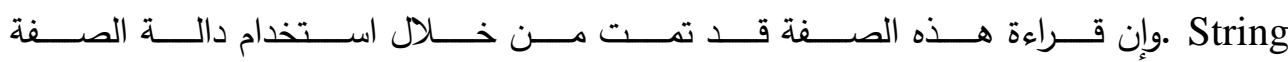
IADsAccessControlEntry :: Object Type

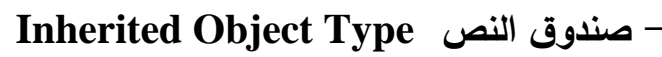
وتثير القيمة فيه إلى نوع كيان الأب لكيان ADSI ـوالقيمة هي القيـ GUID للكيان وبصيغة

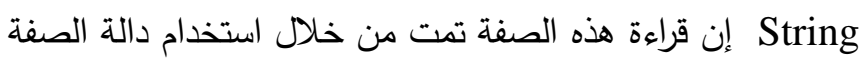
.IADsAccessControlEntry :: InheritedObjectType

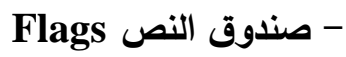
والقيمة فيه تثير إلى فيما إذا كان مدخل التحكم بالوصول يمتلك صنف كيان أم صنف كيان موروث. إن القيم الشرعية لهذه الصفة معرفة في

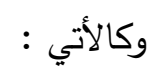

ADS_FLAG_OBGECT_TYPE_PRESENT = 0x1 وتثير إلى وجود حقل ObjectType في مدخل ضبط الوصول ADS_FLAG_INHERITED_OBGECT_TYPE_PRESENT = 0x2 وتثير إلى وجود حقل InheritedObjectType في مدخل ضبط الوصول

Access Mask الإطار وتش 


\section{ويحتوي على مجموعة من ألـ check boxes التي تترجم قناع الوصول إلى سماحات

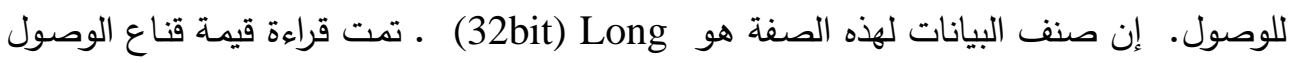

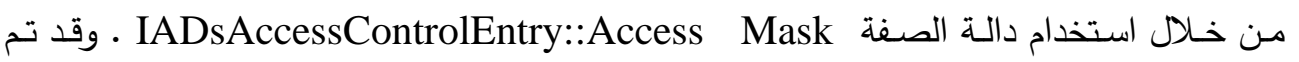 تحليل هذه القيمة إلى bits مفردة لكل منها دلالاتها الخاصة . مله دله}

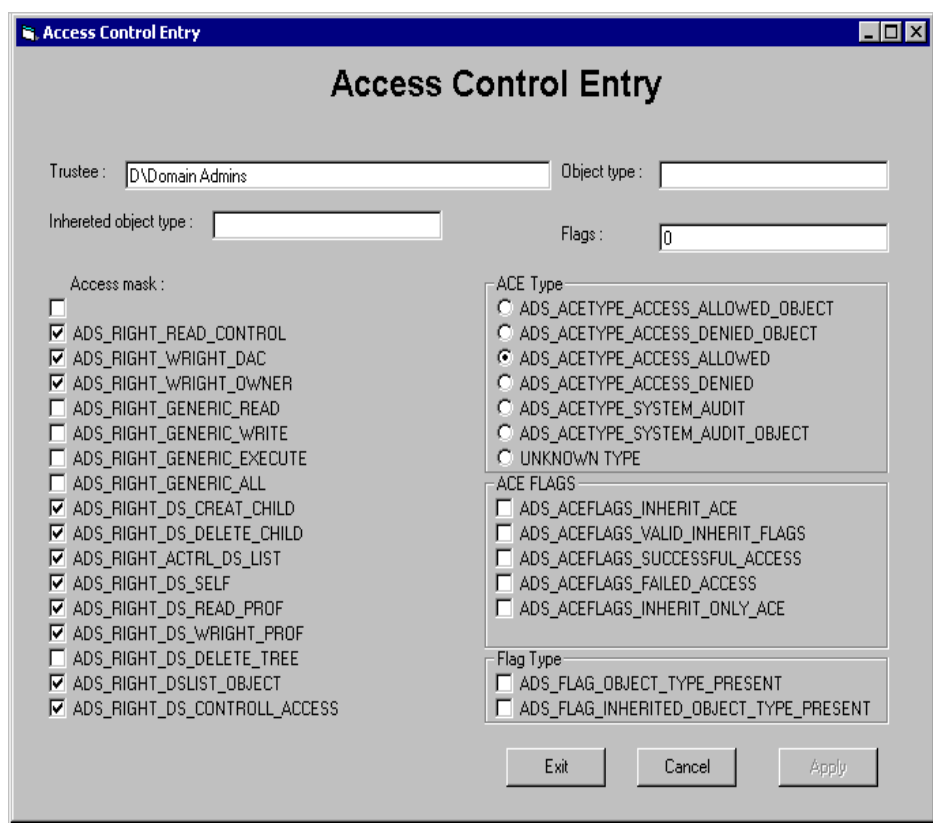

$$
\begin{aligned}
& \text { الثكل(9 ) : واجهة مدخل التحكم بالوصول } \\
& \text { إن مواقع وتسميات أل bits هي : } \\
& \text { • ADS_RIGHT_DELETE=0x10000 } \\
& \text { لمق قراءة المعلومات من الواصف الأمني : ADS_RIGHT_READ_CONTROL=0x20000 } \\
& \text { للكيان ولا يشمل ذلك المعلومات في أل }
\end{aligned}
$$

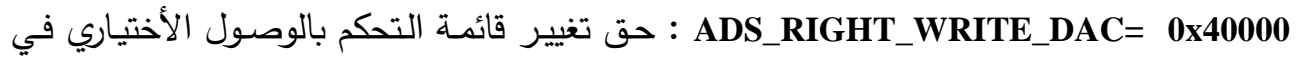

$$
\begin{aligned}
& \text { الواصف الأمني للكيان - الو } \\
& \text { • ADS_RIGHT_WRITE_OWNER=0x80000 } \\
& \text { ADS_RIGHT_ACCESS_SYSTEM_SECURITY= 0x1000000 : حق القراءة من أو تغيير ألـ } \\
& \text { • في الواصف الأمني للكيان SACL }
\end{aligned}
$$


ADS_RIGHT_GENERIC_READ=0x80000000 : حق القراءة من الواصف الأمني للكيان تفحص الكيان وأبنائه وقراءة جميع الصفات .

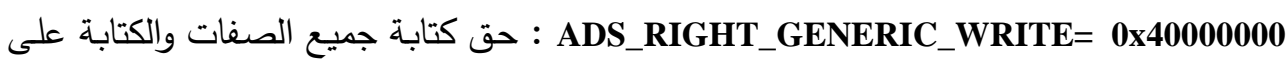
أل أل DACL

• ADS_RIGHT_GENERIC_EXECUTE= 0x20000000 ADS_RIGHT_GENERIC_ALL= 0x10000000 الثانوية ، قراءة الصفات وكتابتها، تفحص الأبناء والكيان نفسه، وإضـافة الكيان الى الدليل أو إزالته منه والقراءة والكتابة بحق موسع • • ADS_RIGHT_DS_CREATE_CHILD=0x1 ADS_RIGHT_DS_DELETE_CHILD=0x2 ADS_RIGHT_ACTRL_DS_LIST=0x4 ADS_RIGHT_DS_SELF= 0x8

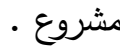
• ADS_RIGHT_DS_READ_PROP=0x10 • ADS_RIGHT_DS_WRITE_PROP=0x20 ADS_RIGHT_DS_DELETE_TREE= 0x40 النظر عن طبيعة السماحات على الأبناء . • ADS_RIGHT_DS_LIST_OBJECT=0x80 ADS_RIGHT_DS_CONTROL_ACCESS=0x100 قبل حق وصول موسع ·

\section{ACE Type الإطار}

وتثير القيمة فيه إلى صنف مدخل التحكم بالوصول ـ إن القيمة هي من صنف بيانات

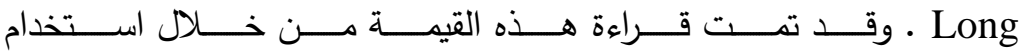

الدالة IADsAccessControlEntry:AceType ،وقد تم تحليل هذه القيمة إلى bits مفردة لكل منها دلالاتها الخاصة .إن قيم هذه الصفة معرفة في ADS _ ACE TYPE_ ENUM وكالاتي 
ADS_ACE TYPE_ACCESS_ALLOWED=0x 0 • ACCESS ALLOWED ADS_ACE TYPE_ACCESS_DENIED= 0x1 · SYSTEM DENIED ADS_ACE TYPE_SYSTEM_AUDIT= 0x2 - القياسي AUDIT وما ADS_ACE TYPE_ACCESS_ALLOWED_OBJECT=0x5 بعده : يمنح مدخل التحكم بالوصول وصولاً إلى كيان ، أو كيان ثانوي من الكيان كمجموعة صفات

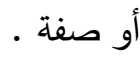

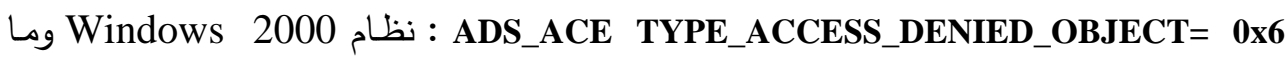
بعده : يمنع مدخل التحكم بالوصول وصولاً إلى كيان ، أو كيان ثانوي من الكيان كمجموعة صفات

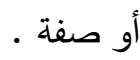
وما ADS_ACE TYPE_SYSTEM_AUDIT_OBJECT= 0x7 بعده : يسجل مدخل التحكم بالوصول ، الوصول إلى كيان أو كيان ثانوي من الكيان مثل مجموعة

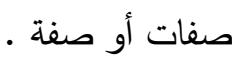

\section{Ace Flag الإطار}

ويحتوي على مجموعـة مـ ألـ check boxes التي تشير قيمها إلى فيما إذا كانت

الكيانات والحاويات الأخرى تستطيع وراثة مدخل التحكم بالوصول من مالك قائمة التحكم بالوصول • تم الحصول على قيم ألـ check boxes من تحليل القيمة المقروءة من خـلال استخدام الدالة IADsAccessControlEntry::Ace Flag

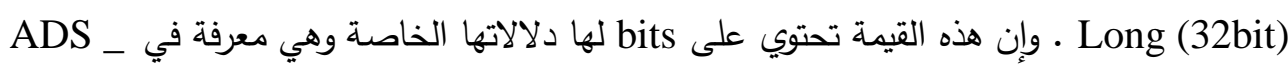
: ACE TYPE _ ENUM

: ADS_ACE_FLAG_INHERIT_ACE= 0x2 ويكون مدخل التحكم بالوصول الموروث قابلاً للوراثة ما لم يكن علماً . 
ADS_ACE FLAG_NO_PROPOGATE_INHERIT_ACE= 0x4 العلم ADS_ACE FLAG_INHERIT_ACE صفراً لمداخل التحكم بالوصـول الموروثة

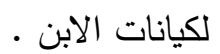

هA ADS_ACE FLAG_INHERIT_ONLY_ACE=0x8 هو لغرض الوراثة فقط ولا يمارس تحكماً في الوصول على الكيان الملحق به. ADS_ACE FLAG_INHERITED_ACE= 0x10 بالوصول موروثاً أم لا . ADS_ACE FLAG_VALID_INHERIT_FLAGS= 0x1F شرعية والنظام هو الذي ينصب هذا أل bit . : ADS_ACE FLAG_SUCCESSFUL_ACCESS= 0x40

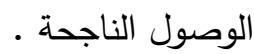
: يولـ رسائل تسجيل لمحساولات الوصـول ADS_ACE FLAG_FAILED_ACCESS= 0x80 الفاشلة .

الاستنتاجات والتوصيات تبين من خلال الدراسة التي تمت في هذا البحث إن هناك محورين رئيسيين للأمنية في

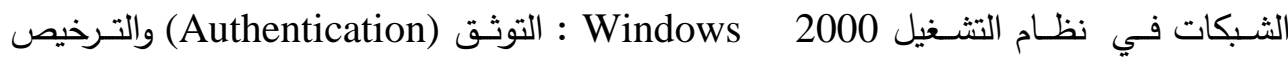
Active وتم التوصل إلى أن هناك خدمات دليل وقاعدة بيانـات هي ألـ (Authorization) تمثل العمود الفقري للعمليات التي تتفذ في سياق هذين المحورين الرئيسين،ولأهمية

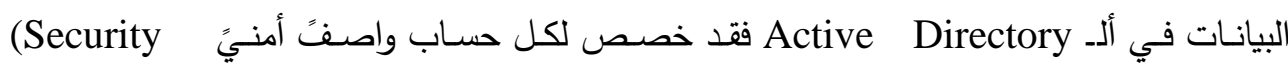
لا يحدد فقط من يمكنه الوصول إلى هذا الحساب ولكن يذهب إلى أبعد من ذلك في

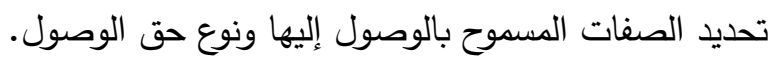
وفي ضـوء المعطيات المذكورة أنفاً تم التوجـه إلى بنـاء برنـامج للسيطرة على حسـابات

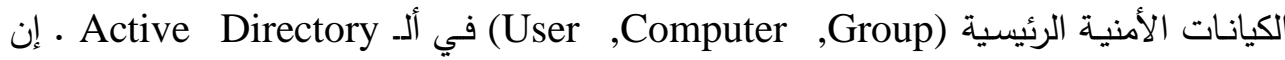
البرنامج هو برنامج للتعامل مع الواصفات الأمنية ومن المستوى الأدنى إذ يذهب في تعامله إلى حد

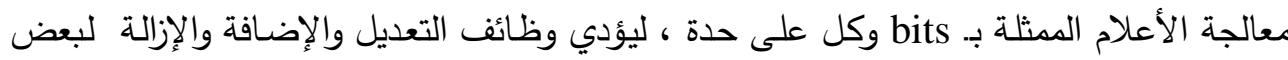

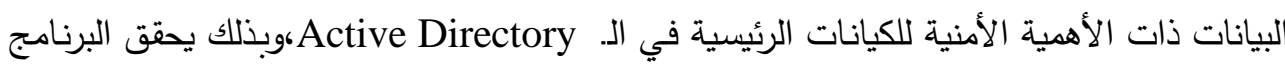

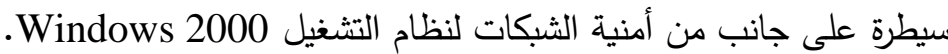


ويمكن الأنطلاق من هذا البرنامج بوصفه عملاً مستقبلياً ليكون نواةً في:

1- تصميم تطبيقات خدمية أمنية خاصة قد تظهر حاجة مدير الدجال إليها.

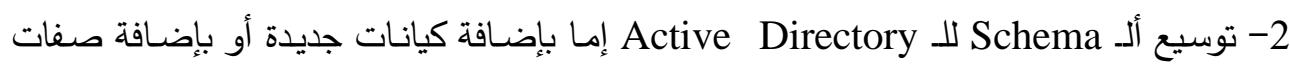

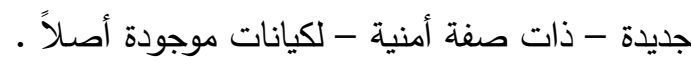

3- توسيع نطاق التعامل مع الكيانات ليتجاوز أل. Active Directory إلى نظام الملفات وإلى ألـ دأل

. Computer registry

4- اقتراح عدد من الحقوق الموسعة الممكنة على الكيانات الموجودة في ألـ Active Directory. تطوير البرنـامج ليفيد في دراسـة الموضوع من خـلال توسيع واجهات البرنـامج بإضـافة عناصر اتصال تعكس التغيير الذي يحدث على البنية التحتية للواصف الأمني بوصفه متغييراً يمكن فهمه دونه من قبل المستخدم العادي . 


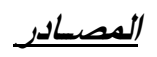

شـلباية، مــراد و فــاروق،علي، 2000 ،مقدمــة الـــى الثـــبكات، دار المسـيرة للنشــر

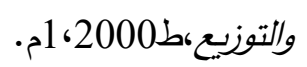

[2] “Active Directory Architecture”, Microsoft, 2001 http://windows.about.com/cs/activedirectory/

[3] Brown K., 2000 , "Exploring Handle Security In Windows", msdn magazine, March 2000 .

[4] Brown K. , 2000 , "Understanding Kerberos Credential Delegation in Windows 2000 Using the TktView Utility", $m s d n$ magazine, May 2000.

[5] Chapil D., 2000, "Exploring Kerberos , The Protocol for Distributed Security in Windows 2000" , MICROSOFT SYSTEM JOURNAL , vol. 4, no. 1, pp40-52.

[6] Esposito D. , 1999 , "With further ADO : Coding Active Directory Data Objects 2.0 with Visual Studio 6.0" , MICROSOFT SYSTEMS JOURNAL, February 1999.

[7] Fox D. , 1999 , "Directory-Enable Apps With ADSI" , VBPJ , February 1999 .

www.devx.com/premier/mgznarch/vbpj/1999/02feb99/fox099.pdf

[8] Grant J. R. , 1999 , “Operating System Security”, April 1997.

http://people.msoe.edu/ sebern/courses/cs384/papers97/grant.pdf

[9] Johansson J. M. , "Windows 2000 Security - An Overview and Analysis. "www.foo.be/docs/adsi-ldap/Windows2000SecurityAduserpasswordproperties.pdf

[10] “Kerberos in Win2K", Windows NT magazine, October 1999.

[11] Kling J. , 1999 , Working with Objects in Active Directory , Exploring Windows NT, December 1999.

[12] Mrozowski P. , 2001, "Taking Advantage of ADSI" , CoDe magazine, Winter 2001.

www.kirtlandsys.com/misc/Taking\%20Advantage\%20of\%20ADSI. $\underline{p d f}$

[13] Otel F.,2000 , "Some Security Aspects of Link Layer Protocol”, www.ce.chalmers.se 
[14] Waddell J. L. , 2001 , "Basic Security Issues of Active Directory", SANS Institute, June 2001 .

www.set.usn.edu/SBlesse/ref/docs/MICROSOFT-

Basic\%20Security\%20Issues\%20of\%20Active\%20Directory.pdf

[15] Wildermuth S. , 2000 , “Active Directory Doesn't Manage Network Resources, It Can Manage Your Data Too", MICROSOFT SYSTEM JOURNAL, January 2000. 